\title{
Mitochondrial DNA deletion diagnosed by analysis of an endomyocardial biopsy specimen from a patient with Kearns-Sayre syndrome and complete heart block
}

\author{
Anne M Remes, Ilmo E Hassinen, Kari Majamaa, Keijo J Peuhkurinen
}

\begin{abstract}
Defects of mitochondrial DNA have been found at necropsy in the myocardium of patients with Kearns-Sayre syndrome. A patient with characteristics typical of Kearns-Sayre syndrome and a complete heart block is described. Southern blot analysis showed a deletion of $3.3 \mathrm{~kb}$ in the mitochondrial DNA in an endomyocardial biopsy specimen and in skeletal muscle. The deletion led to the disappearance of the genes for four transfer RNAs and four subunits of complex I (NADH:ubiquinone oxidoreductase) in the mitochondrial respiratory chain. The defect could not be demonstrated in whole blood despite amplification of the mitochondrial DNA region of interest by the polymerase chain reaction technique. There can be heteroplasmy-that is, normal and abnormal mitochondrial DNA populations in one cell-in different tissues, and the degree of heteroplasmy may be crucial in the development of organ-specific symptoms. This patient raises the possibility that some tissues can be specifically enriched with mitochondria with DNA defects and emphasises the need for elective sampling of the target tissue and polymerase chain reaction technique to exclude these defects. The role of mitochondrial DNA defects in idiopathic cardiomyopathies could perhaps be studied by analysis of mitochondrial DNA from endomyocardial biopsy specimens.
\end{abstract}

\section{(Br Heart J 1992;68:408-11)}

Kearns-Sayre syndrome is characterised by onset before age 20, progressive external ophthalmoplegia, and abnormal retinal pigmentation. In addition, diagnosis depends on at least one of the following signs being present: complete heart block, cerebellar dysfunction, or high cerebrospinal fluid protein. ${ }^{1}$ The syndrome belongs to the category of mitochondriopathies with defects in mitochondrial $\mathrm{DNA}^{2}$ and it has been suggested that selective involvement of the cardiac conduction system leads to conduction abnormalities. ${ }^{3-5}$ A patient with the typical characteristics of KearnsSayre syndrome and rapid development of congestive heart failure who required heart , transplantation has been reported, however. ${ }^{6}$

Mitochondrial DNA is a small $(16.5 \mathrm{~kb})$, circular, double stranded molecule that contains 13 genes for peptides of the mitochondrial respiratory chain, 12 transfer RNA genes, and two genes encoding mitochondrial ribosomal RNAs. ${ }^{2}$ The diagnosis of cardiac involvement in mitochondriopathies has relied on typical electrocardiographic changes and ultrastructural findings in an endomyocardial biopsy specimen ${ }^{7}$ or the analysis of mitochondrial DNA in hearts at necropsy. ${ }^{89} \mathrm{We}$ describe a patient presenting with a complete heart block and clinical features of KearnsSayre syndrome, in whom the mitochondrial DNA defect was characterised and its distribution in various tissues studied.

\section{Case report}

The patient had an uneventful childhood, but noticed weakness of the eyelids at the age of 17; one year later bilateral ptosis was seen. Retinal degeneration and decreased visual acuity were also diagnosed, but ocular movements were intact. A neurological examination at the age of 18 showed myopathic facies, diffuse slowing in the electroencephalogram, normal cerebrospinal fluid protein (330 $\mathrm{mg} / \mathrm{l})$, and a normal electromyogram. Symmetrical hearing loss was continued at the age of 19 and ocular movements were restricted in all directions at 21 . Several operations were performed later for bilateral ptosis, and in his 30's he began to experience muscle weakness during exercise, although he was still able to work on the family farm.

The patient had no cardiac symptoms until he collapsed on two occasions at the age of 38 , whereupon a complete electrocardiographic heart block was diagnosed. An electrophysiological examination showed a block in the conduction system distal to the His bundle (fig 1), and a DDD pacemaker was implanted. Echocardiography was normal.

A neurological examination showed a general decrease in muscle mass and specifically muscle atrophy and weakness of the facio- 
Figure 1

Electrocardiogram showing conduction block distal to His bundle. Upper and middle tracings are lead $V 2$ in 1981 and 1982 respectively. Lower tracing is His

electrocardiogram in 1991 $A$, atrial deflection; $\mathrm{H}, \mathrm{His}$ potential; $P$, pacemaker artefact; $V$, ventricular complex.
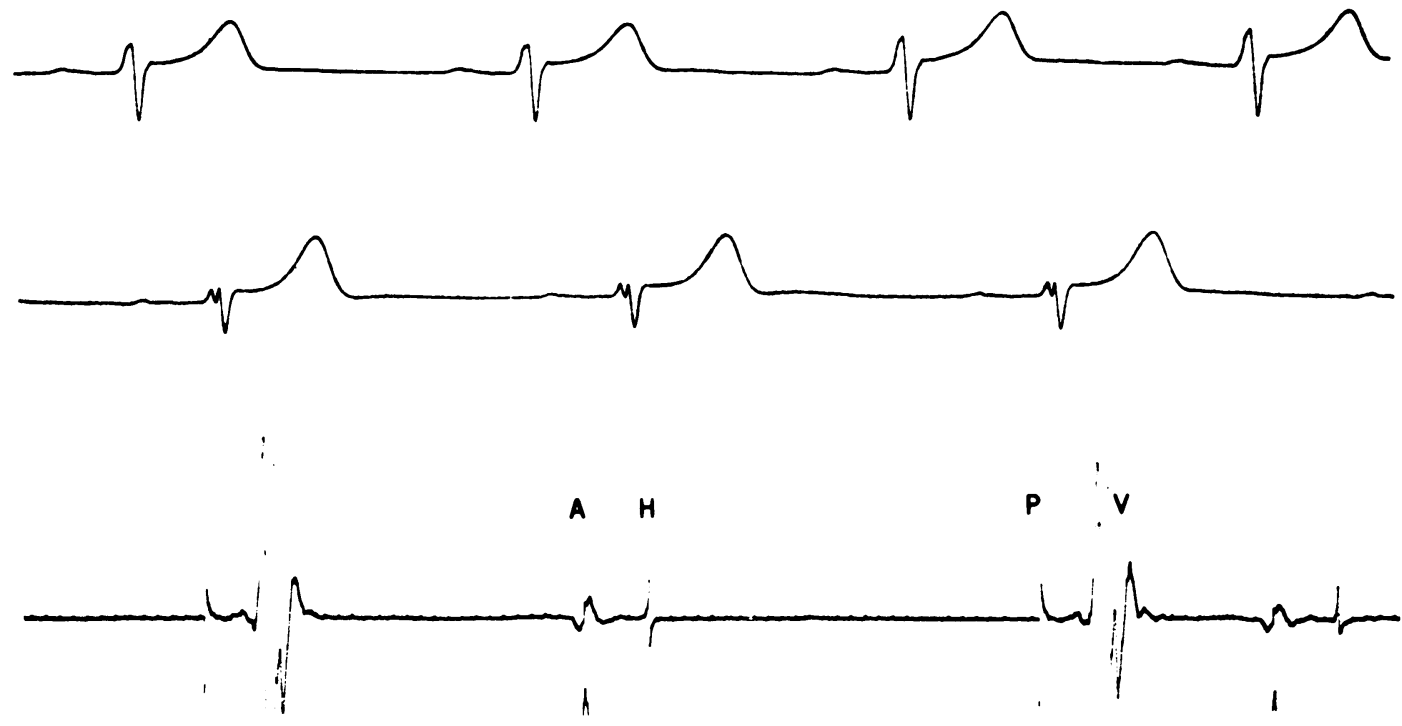

scapulohumeral type. There was a complete external ophthalmoplegy, moderate bilateral ptosis and pigmentary retinopathy. Tendon reflexes, sensation, and coordination were normal. The electroencephalogram was diffusely abnormal, with theta activity. Computed

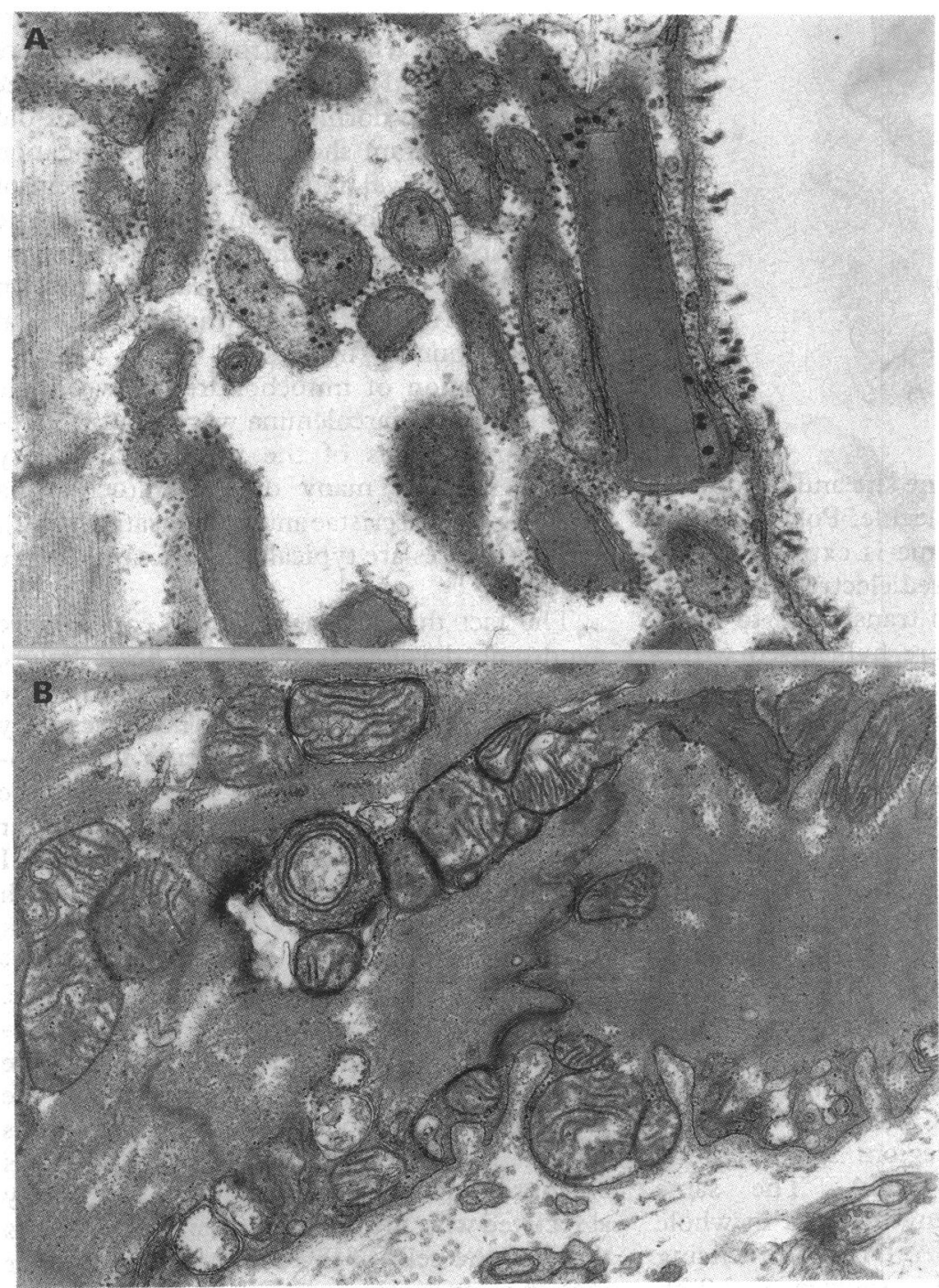

Figure 2 (A) Electron micrograph of skeletal muscle showing abnormal mitochondria with paracrystalline inclusions and a concentric arrangement of the cristae.

$(B)$ Electron micrograph of endomyocardium showing prominent accumulations of mitochondria, some of which showed concentric cristae. brain tomography showed cortical atrophy. Nerve conduction velocities were normal but the electromyogram showed changes consistent with mild myopathy. There was a symmetrical sensorineural hearing loss. He was considered to be of average intelligence.

There were no abnormalities in routine laboratory tests and no raised titres of antibodies to any micro-organism were detected. Antinuclear antibodies (IgG) were somewhat raised as was the resting blood pyruvate concentration (115 $\mu \mathrm{mol} / \mathrm{l}$, normal range 45-85). The resting lactate concentration varied from 1.31 to $2.80 \mathrm{mmol} / \mathrm{l}$ (normal range $1 \cdot 0-1 \cdot 8)$, and during a forearm ischaemic exercise test lasting two minutes (five-fold increase normal) increased $7 \cdot 5$ fold from the baseline value. The urine aminoacid analysis was normal.

Several endomyocardial biopsy specimens and a skeletal muscle biopsy specimen were taken for histopathological examination and mitochondrial DNA analysis. Light microscopy after Gomori trichrome staining of the skeletal muscle showed scattered ragged red fibres but otherwise the muscle was normal. Electron microscopy study showed abnormal mitochondria with paracrystalline inclusions and a concentric arrangement of the cristae (fig 2A). The endomyocardium was normal by light microscopy, but electron microscopy showed prominent accumulations of mitochondria, some of which showed abnormal concentric cristae (fig 2B).

The activities of the mitochondrial respiratory chain enzymes were analysed by oximetry and spectrophotometric methods applied to mitochondria isolated from the skeletal muscle. The activities of the enzymes were normal when studied by oximetry, but spectrophotometry showed a decrease in NADH-cytochrome $c$ reductase activity (42 $\mathrm{nmol} / \mathrm{min} / \mathrm{mg}$ protein, normal range 63-230). Total DNA was isolated from frozen endomyocardial and skeletal muscle biopsy specimens and whole blood by the standard phenol/chloroform/isoamyalcohol method $1 \mu \mathrm{g}$ of DNA was digested with the restriction enzyme Pvu II, which cuts the mitochondrial 
Figure 3 Southern blot analysis of blood $(B)$, heart $(H)$, and skeletal muscle (M) from a patient with Kearns-Sayre syndrome. $C$, control.

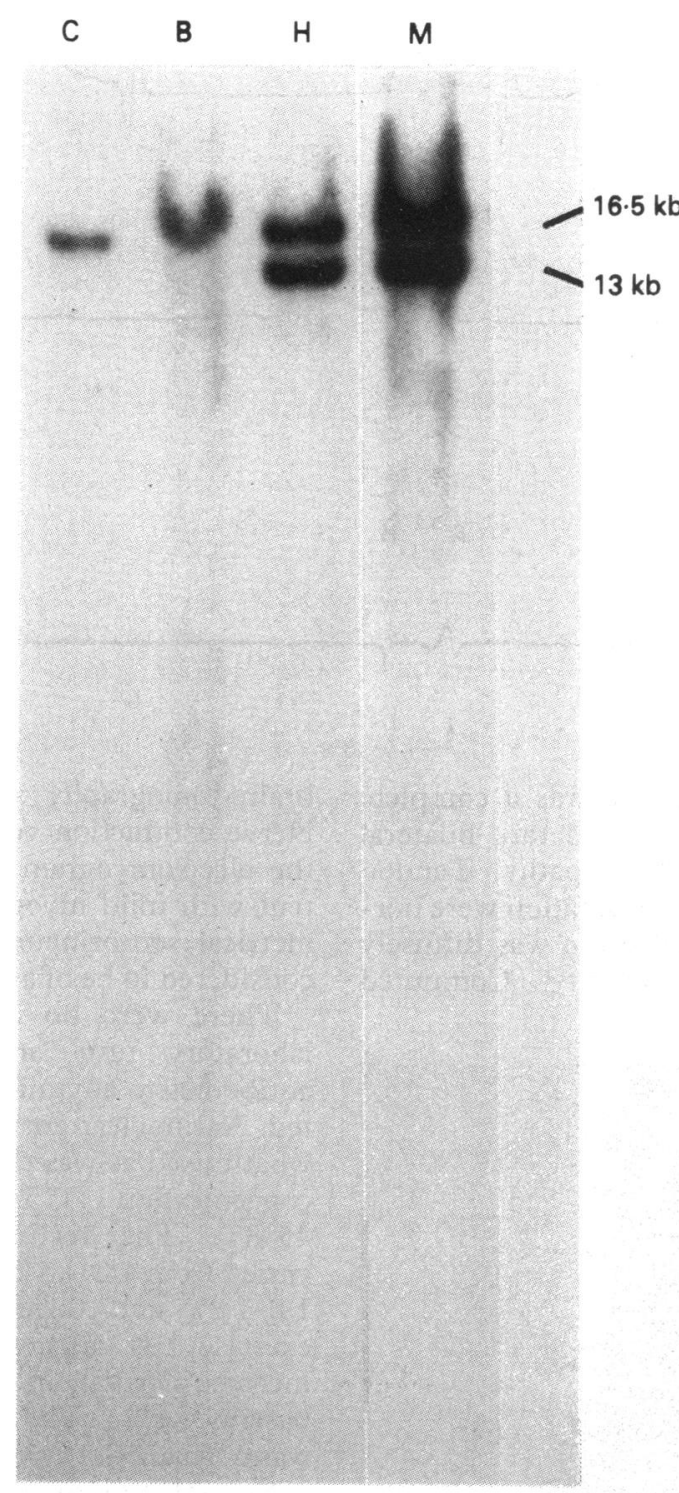

DNA molecule at only one site and produces a single $16.5 \mathrm{~kb}$ linear molecule. Polymorphism for this restriction enzyme is extremely rare. The digests were separated electrophoretically in $0.8 \%$ agarose gel and transferred to nitrocellulose. The filters were hybridised with a whole mitochondrial DNA probe isolated from human placenta and radiolabelled by the random primer method. Southern blot analysis of heart and skeletal muscle (but not blood) showed two populations of mitochondrial DNA, one corresponding to full-length molecules of mitochondrial DNA and the other, which migrated more rapidly in the electrophoretic gel, corresponding to a smaller mitochondrial DNA (fig 3). The deleted portion of the mtDNA was roughly located by analysis for the absence of known restriction sites. $^{2}$ After mapping, the polymerase chain reaction technique was used for selective amplification of the region spanning the deleted mitochondrial DNA. The same method did not detect any deletion in whole blood mitochondrial DNA. Direct sequencing of the amplified product showed a $3.3 \mathrm{~kb}$ deletion (map position 10169-13406 in the Cambridge sequence). The deleted region included four transfer RNAs (Arg, His, Ser,
Leu) and four subunits of Complex I of the mitochondrial respiratory chain.

\section{Discussion}

The cardiac abnormalities associated with the Kearns-Sayre syndrome have been considered to be limited to the conduction system. The most common conduction abnormality is left anterior hemiblock, alone or in combination with a right bundle branch block; while others include Mobitz type II second degree atrioventricular and complete heart block. ${ }^{4-6}$ Progressive impairment of infranodal conduction is typical of these patients, but, somewhat paradoxically, increased velocity of atrioventricular nodal conduction has also been seen. ${ }^{4}$ Because of the progressive nature of the conduction abnormality and the increased life-threatening risk associated with high-degree infranodal heart block, prophylactic pacemaker treatment has been recommended for these patients. ${ }^{410} \mathrm{It}$ is important to differentiate between KearnsSayre patients and patients having left axis deviation in combination with a right bundle branch block without myocardial infarction, because the latter do not generally require pacemaker therapy. ${ }^{11}$

Our patient presented with a complete heart block. Two earlier electrocardiograms were available (10 and nine years old). The older one was apparently normal, but the nine year old electrocardiogram showed some prolongation of the PR interval and signs of impairment of intraventricular conduction, confirming the progressive nature of the conduction abnormality. The electrophysiological examination showed that the block was infranodal and distal to the His bundle (fig 1 ).

Proliferation of mitochondria between the myofibrils and sarcolemma was found in electron micrographs of the myocardial biopsy specimen, with many of the mitochondria showing curved cristae and vacuolisation (fig 2). These features are typical of the Kearns-Sayre syndrome. ${ }^{10}$

The fact that a patient with Kearns-Sayre syndrome developed congestive heart failure requiring transplantation ${ }^{6}$ suggests that the defect in mitochondrial DNA is not necessarily limited to the conduction system. The mitochondrial DNA deletion of $3.3 \mathrm{~kb}$ found in the present case leads to defective synthesis of four transfer RNAs and four subunits of Complex I in the mitochondrial respiratory chain, which should impair oxidative energy transfer. Mechanical function, as assessed by echocardiography, was perfectly normal, however. This finding also accords with measured mitochondrial enzyme activities and points to the fact that a considerable proportion of the heteroplasmic mitochondrial population is normal. It may also be that the cardiomyocytes are more resistant to the metabolic abnormality associated with the mitochondrial DNA defects than are the cells of the conduction system or that the conduction system is enriched with mitochondria with DNA deletions. On the other hand, the amount of deleted mitochondrial DNA in different tissues and organs-that 
is, the degree of heteroplasmy-may determine the final manifestations of the syndrome. ${ }^{12}$

It has been recognised that diseases related to defects in mitochondrial DNA may be tissue specific, and the dogma of identical mitochondrial DNA in all the cells of an individual no longer holds true. This was also apparent in our patient, in whom heteroplasmy was shown in the tissues related to the symptoms but not in the leucocytes or platelets, for example. A skeletal muscle biopsy may therefore not be sufficient to diagnose or exclude cardiomyopathy caused by defective mitochondrial DNA.

Deletion of mitochondrial DNA is commonly detected in elderly subjects, the proportion of the deleted species increasing with age. Moreover, it has been suggested that accumulation of this deletion may contribute to the ageing process and the occurrence of presbycardia. $^{13}$

It has been thought that the aetiology of dilated cardiomyopathy is related to autoimmunity, suggesting that a predisposition towards it may be determinated by the major histocompatibility complex genes, ${ }^{14}$ but a viral aetiology has also been reported. ${ }^{15}$ It may well be that dilated cardiomyopathy is multifactorial in origin, as some cases of isolated cardiomyopathy have been recently described in which there were multiple mitochondrial DNA deletions or point mutations. ${ }^{81617}$ This emphasises the role of organ-specific sampling and analysis of mitochondrial DNA. A prospective study to search for mitochondrial DNA defects may help to clarify the role of these genetic defects-for example, in patients with dilated cardiomyopathy.

We thank Dr Heikki Huikuri for performing the electrophysiological examination, Dr Riitta Herva for analysing the histological samples, and Ms Anja Roukala for technical assistance with the DNA analysis.
This study was supported by the Sidrig Juselius Foundation and the Medical Research Council of the Academy of Finland.

1 Pavlakis SG, Rowland LP, DeVivo DC, et al. Mitochondria myopathies. In: Plum F, ed. Advances in contemporary neurology. Philadelphia: Davis, 1988:95-133.

2 Zeviani M, Moraes CT, Di Mauro S, et al. Deletions of mitochondrial DNA in Kearns-Sayre syndrome. Neurology 1988;38:1339-46.

3 Uppal SC. Kearns' syndrome, a new form of cardiomyopathy. Br Heart J 1973;35:766-9.

4 Roberts NK, Perloff JK, Kark RAP. Cardiac conduction in the Kearns-Sayre syndrome (A neuromuscular disorder associated with progressive external ophthalmoplegia and pigmentary retinopathy). Am J Cardiol 1979;44: $1396-400$.

5 Kenny D, Wetherbee J. Kearns-Sayre syndrome in the elderly: Mitochondrial myopathy with advanced heart block. Am Heart J 1990;120:440-3.

6 Channer KS, Channer JL, Campbell MJ, Russel Rees J. Cardiomyopathy in the Kearns-Sayre syndrome. $B r$ Heart J 1988;59:486-90.

7 Schwartzkopff B, Frenzel H, Breithardt G, et al. Ultrastructural findings in endomyocardial biopsy of patients with Kearns-Sayre syndrome. J Am Coll Cardiol 1988;12: 1522-8

8 Ozawa T, Tanaka M, Sugiyama SY, et al. Multiple mitochondrial deletions exist in cardiomyocytes of patients with hypertrophic or dilated cardiomyopathy. Biochem Biophys Res Commun 1990;170:830-6.

9 Zeviani M, Gellera C, Antozzi C, et al. Maternally inherited myopathy and cardiomyopathy: association with mutation in mitochondrial DNA tRNA Leu (UUR). Lancet 1991; 338:143-7.

10 Charles R, Holt S, Kay JM, Epstein EJ, Russel Rees J. Myocardial ultrastructure and the development of atrioventricular block in Kearns-Sayre syndrome. Circulation 1981;63:214-9.

11. Kulbertus HE. Reevaluation of the prognosis of patients with LAD-RBBB. Am Heart $J$ 1976;92:665-7.

12 Zeviani M, Gellera C, Pannacci M, et al. Tissue distribution and transmission of mitochondrial DNA deletions in mitochondrial myopathies. Ann Neurol 1990;28:94-7.

13 Hattori K, Tanaka M, Sugiyama S, et al. Age-dependent increase in deleted mitochondrial DNA in the human heart: possible contributory factor to presbycardia. $A m$ Heart $J$ 1991;121:1735-42.

14 Carlquist JF, Menlove RL, Murray MB, O'Connel JB, Anderson JL. HLA class II (DR and DQ) antigen associations in idiopathic dilated cardiomyopathy: Validation study and meta-analysis of published HLA association studies. Circulation 1991;83:515-22.

15 Bender JR. Idiopathic dilated cardiomyopathy: An immunologic, genetic, or infectious disease, or all of the above. Circulation 1991;83:704-6.

16 Tanaka M, Ino H, Ohno K, et al. Mitochondrial mutation in infantile cardiomyopathy. Lancet 1990;336:1452.

17 Ozawa T, Tanaka M, Sugiyama S, et al. Patients with idiopathic cardiomyopathy belong to the same mitochondrial DNA gene family of Parkinson's disease and mitochondrial encephalomyopathy. Biochem Biophys Res Commun 1991;177:518-25. 